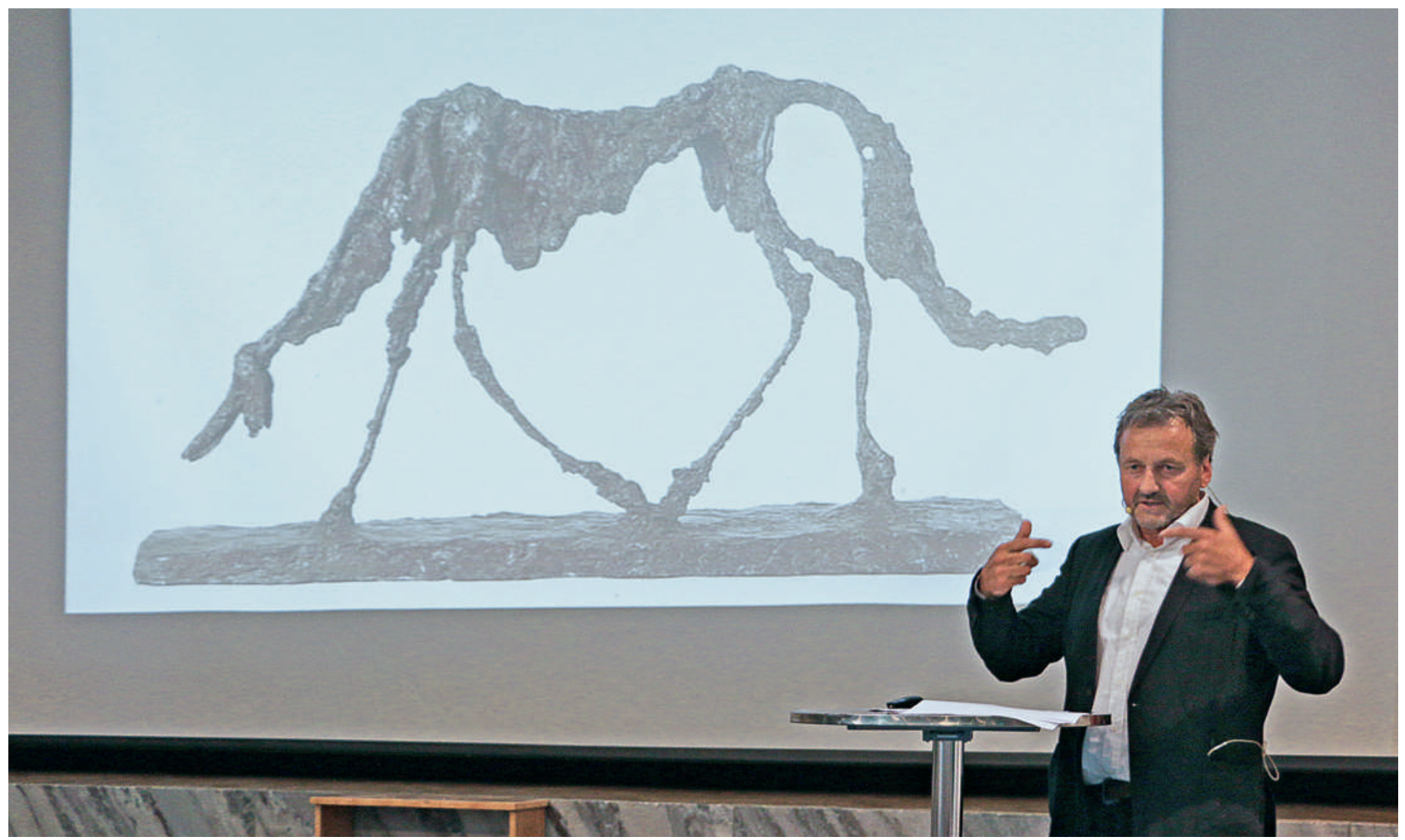

"Gutes Bauen macht gesund oder zumindest nicht krank»: Der Architekt Gion A. Caminada bei seinem Vortrag, in dem er auch über die Skulptur Der Hund seines Bündner Landsmanns Alberto Giacometti sprach.

\title{
Segen mit Schattenseite
}

\section{Bruno Kesseli (Text und Bilder)}

Dr. med. et lic. phil., Chefredaktor

Der technische Fortschritt hat der Medizin ungeahnte Möglichkeiten eröffnet. Eine einseitig auf die Technik ausgerichtete Medizin wird dem Menschen aber nicht gerecht, und ihr Potential verringert sich. Dieses kann nur durch ein Verständnis der Medizin als umfassende Heilkunst ausgeschöpft werden. Die zentralen Aussagen des Symposiums «Technik - Mensch - Heilkunst» waren nicht neu, wurden aber in origineller und anregender Form präsentiert.

Viele Symposien kokettieren damit, «anders» zu sein die von der Akademie Menschenmedizin organisierte Tagung «Technik - Mensch - Heilkunst» war es. Und dies in mehrfacher Hinsicht. So hatte die Kunst an diesem Anlass nicht einfach den Stellenwert von nettem 'Beigemüse', sondern war integrierender Bestandteil der Veranstaltung. Die Referate wurden vom Pianisten André Desponds unmittelbar nach Abschluss virtuos musikalisch kommentiert und fanden damit einen künstlerisch-sinnlichen Nachklang, der vom Publikum sehr positiv aufgenommen wurde. So positiv, dass man sich des Eindrucks nicht erwehren konnte, der Verzicht auf intellektualisierende Fragerunden oder Diskussionen, die auch die Gefahr des 'Zerredens' in sich bergen, werde weniger als Verlust denn als Wohltat erlebt. 


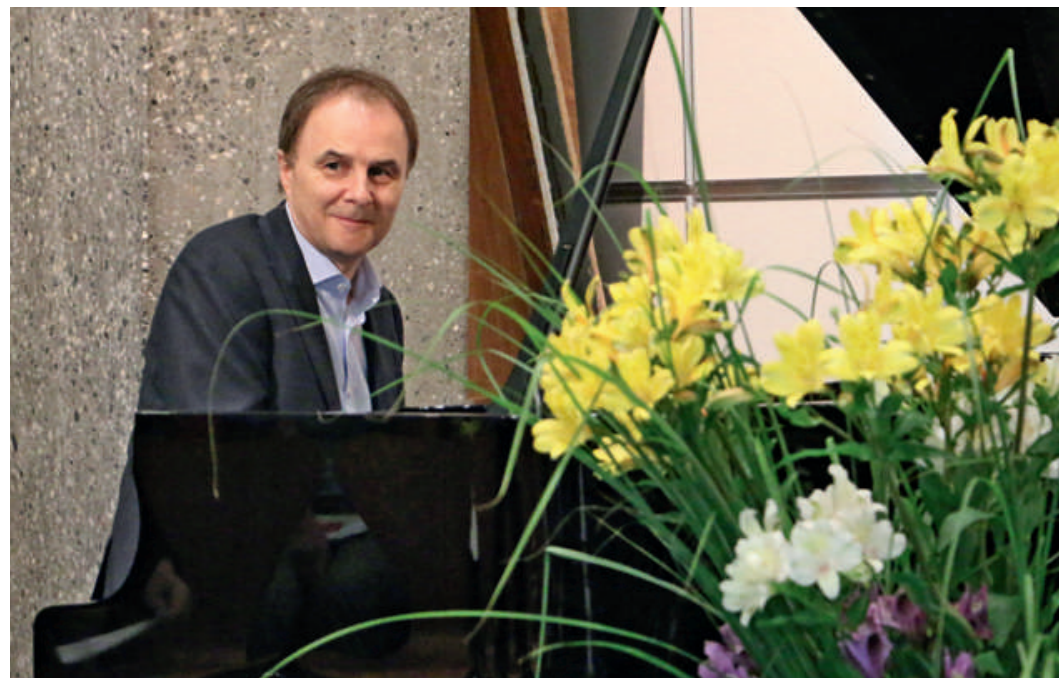

Der Pianist André Desponds kommentierte die einzelnen Referate musikalisch.

\section{Weit gefasster Rahmen}

Neben dem Pianisten Desponds erhielt die Medienkünstlerin Chantal Michel viel Raum für Präsentationen und Interventionen. Und schliesslich trat auch der Vorstand der Akademie Menschenmedizin mit zwei «Intermezzi» aktiv in Erscheinung. In einer Form, die an die Funktion des Chors in den klassischen griechischen Dramen erinnerte, wurde das Verhältnis von Kunst und Heilkunst reflektiert, kommentiert und kritisch hinterfragt. Eine bemerkenswerte und im heutigen Kongressbetrieb aussergewöhnliche Eigenleistung, die dem Engagement der Organisatoren für die von ihnen postulierte «Menschenmedizin» Glaubwürdigkeit verlieh.

Auch was die Auswahl der Referentinnen und Referenten betrifft, war das Bemühen spürbar, den üblichen Rahmen wenn nicht gerade zu sprengen, so zumindest weit zu fassen. Neben Medizinern wie dem Herzchirurgen Thierry Carrel und dem Hausarzt Louis Litschgi und Fachpersonen aus dem Pflegebereich wie Schwes-

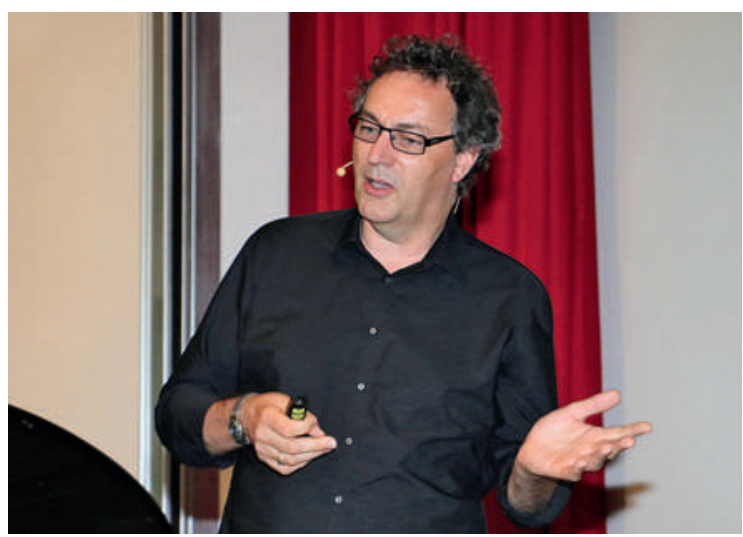

Gerd Leonard. ter Liliane Juchli und Pflegewissenschaftlerin Rebecca Spirig kamen auch Persönlichkeiten mit weniger direktem Draht zur Medizin zu Wort. In diese Kategorie fielen der Futurist und Zukunftsberater Gerd Leonard, der Architekt Gion A. Caminada und der Elektroingenieur Anton Gunzinger, bekannt unter anderem als Entwickler eines superschnellen Computers. Eine Mischung, die sowohl thematisch als auch 'weltanschaulich' für Abwechslung und Spannung sorgte.

\section{Enhancement à discretion?}

Bereits der Vortrag von Gerd Leonard, der als einer der ersten auftrat, machte die Ambivalenz deutlich, die viele angesichts der in schwindelerregendem Tempo fortschreitenden technischen Entwicklungen empfinden, insbesondere wenn diese die geistigen Funktionen des Menschen betreffen. Technikvisionäre wie Ray Kurzweil scheinen das Verschmelzen von Mensch und Maschine, vielleicht sogar die Überwindung des Menschseins, wie wir es bisher kannten, kaum erwarten zu können. Was sie sich genau davon versprechen, ist nicht ganz klar. Wird unser Leben dadurch sinnvoller? Werden wir glücklicher? Oder wird der in der amerikanischen Verfassung festgeschriebene «Pursuit of happiness» dadurch nicht einfach auf eine Ebene verlagert, auf der genau das gleiche Strampeln wie bisher abläuft, nur dass man ohne implantierte Chips und weitere Enhancement-Tools nicht mehr wird mithalten können? Werden sich dadurch die Chancen für Unterprivilegierte verbessern, oder wird die Kluft zu den Privilegierten, die sich Enhancement à discretion leisten können, nicht noch unüberbrückbarer werden?

Gemäss Leonard ist die Konvergenz von Mensch und Maschine bereits im Gang - Hunderte von Millionen würden darin investiert. Das Internet bezeichnet er als neue Religion, Smartphones als neue Zigaretten, die uns genauso beherrschen wie wir sie. Die Frage, wie ein

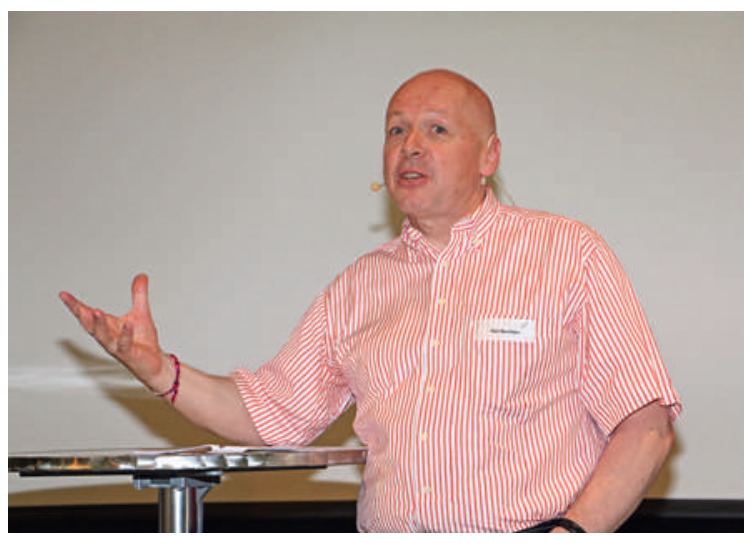

Anton Gunzinger. 
guter Umgang mit diesen Tendenzen aussehen könnte, beantwortet auch der Futurist interessanterweise mit «aktive Begrenzung», Neudeutsch "Self Limiting». Man solle den Unterschied feiern, zwischen virtueller Welt und echter Erfahrung: «Stop looking at your phone look around you.» Bei allem Segen, den der technische Fortschritt der Menschheit gebracht hat, scheint eine grenzenlose Technik-Euphorie nicht flächendeckend um sich zu greifen. Zumindest wächst offenbar die Zahl derer, die dem technischen Fortschritt grundsätzlich positiv gegenüberstehen und moderne Technik gerne nutzen, sich aber aus einem vielleicht urmenschlichen Freiheits- und Unabhängigkeitsdrang in gewissem Mass davon emanzipieren wollen.

\section{Störfall Patient}

Diese duale Sichtweise in Bezug auf die Technik kam auch in den Referaten der Ärzte Louis Litschgi und Thierry Carrel sowie der Pflegewissenschaftlerin Rebecca Spirig zum Ausdruck. Die Technik gehöre zwar zum Menschen, wurde betont, aber jedes Leben sei einzigartig, und es sei illusionär zu glauben, dass mit dem technischen Fortschritt alle Probleme gelöst werden könnten. Im ärztlichen Bereich könne die Apparatemedizin auch zu einer Bedrohung für die Beziehungsmedizin werden. Im Krankheitsfall wünschten wir uns aber eben genau dies, eine Beziehung, die Begleitung durch eine Person, die uns versteht. Sehr radikal formulierte dies die Ordensschwester und Pflegefachfrau Liliane Juchli, die durch ihr Pflegemodell und ein von

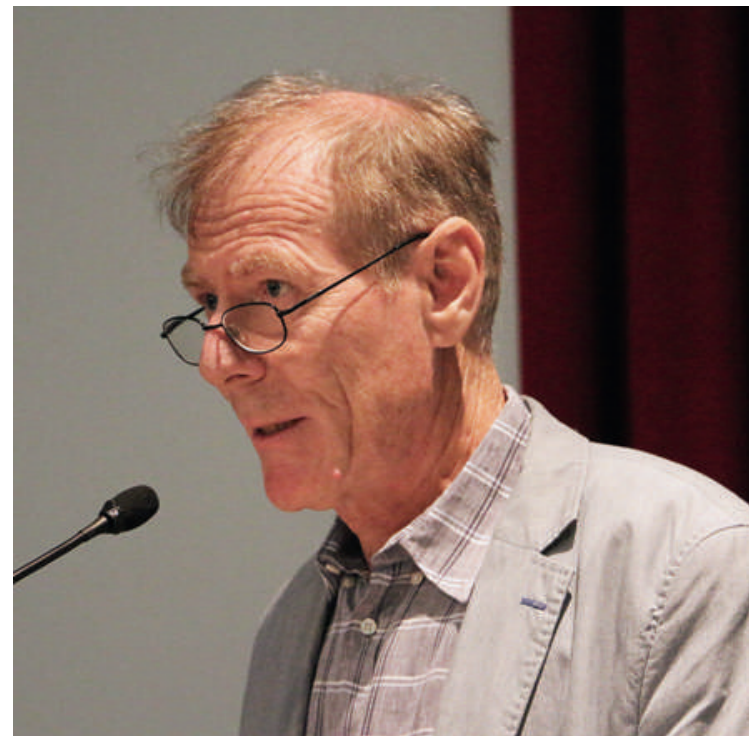

Louis Litschgi.

ihr verfasstes, zum Standardwerk gewordenes Pflegelehrbuch, einen grossen Bekanntheitsgrad erfahren hat. Mit 81 Jahren quicklebendig, mit Schlagfertigkeit und Humor gesegnet, fragte sie kritisch, ob der Mensch im heutigen Gesundheitssystem tatsächlich im Mittelpunkt stehe, wie dies so gerne und oft postuliert werde, oder ob der Patient nicht eher zum Störfall geworden sei. Pflegenden werde es zunehmend schwer gemacht, ihre Vorstellungen von menschengerechter Betreuung der Patienten umzusetzen. Das Geheimnis der Pflege lässt sich nach Juchli in einem Wort zusammenfassen: Präsenz.

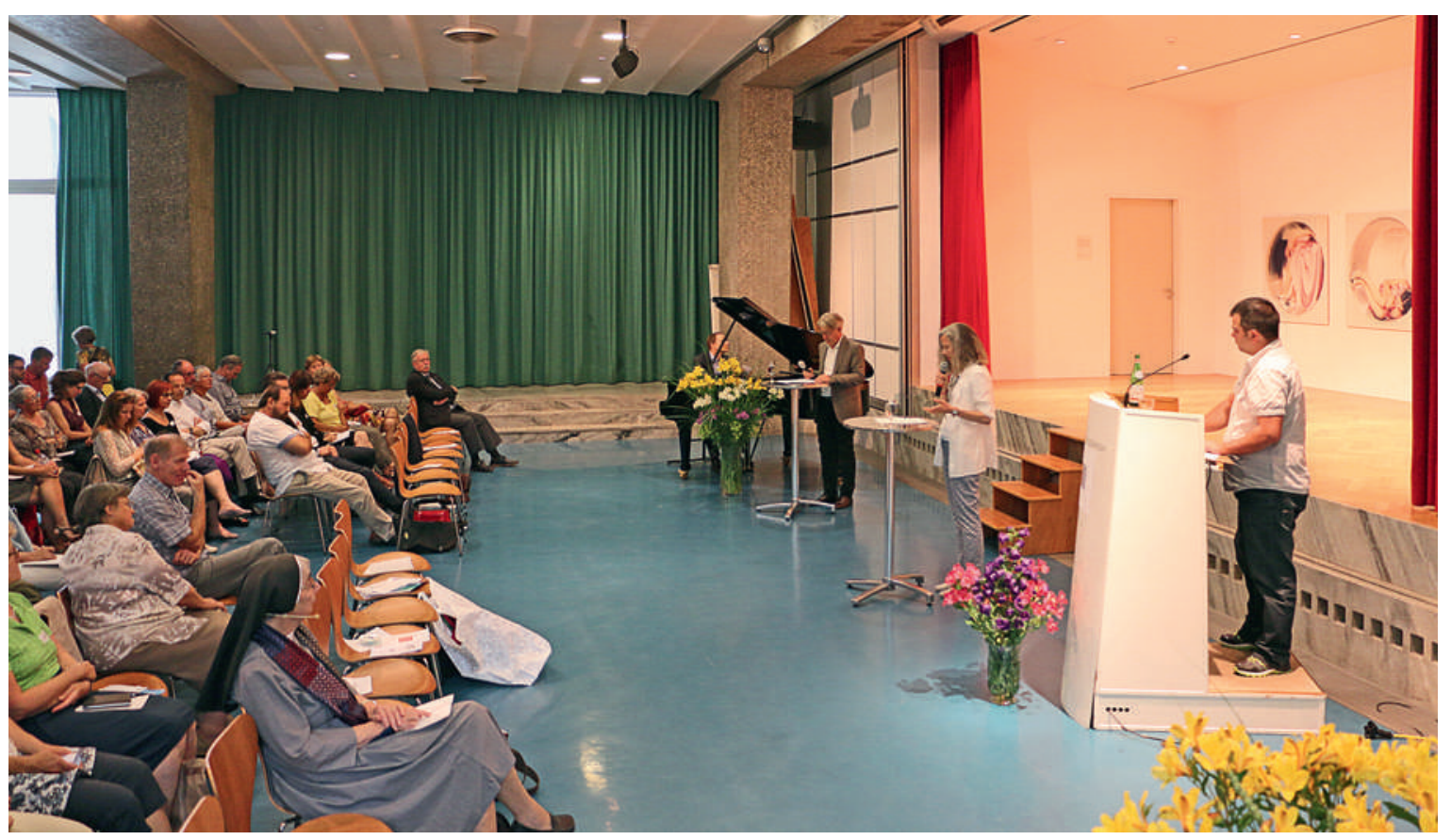

Vorstandsmitglieder der Akademie diskutierten in «Intermezzi» das Verhältnis von Kunst und Heilkunst. 


\section{Alles zu Gold machen}

Der Begriff der Präsenz charakterisierte auch den Auftritt des Architekten Gion A. Caminada treffend. Caminada, der in Vrin ein Architekturbüro betreibt und als Professor für Architektur und Entwurf an der ETH Zürich lehrt, sprach mit Eindringlichkeit über seine Beziehung zu Natur und Technik und die einzigartige Schönheit von Kuhaugen («die schönsten Augen aller Lebewesen»). Bei seiner Lehrtätigkeit ist es ihm ein wichtiges Anliegen, die Autonomie der Studentinnen und Studenten zu stärken. Sie sollen selbstbewusst sein, ohne darüber die Solidarität zu vergessen. Einen wichtigen Bezugspunkt zwischen Architektur und Heilkunst sieht Caminada darin, dass «gutes Bauen gesund macht, oder zumindest nicht krank». Die Technik bezeichnet er als «etwas Grossartiges, aber sie ist nicht nur ein Segen». Die einseitige Ausrichtung auf die Technik könne Kulturen zerstören. Sich selbst nimmt er im Umgang mit der Technik als Alchimisten wahr: «Der Alchimist will alles zu Gold machen.» Die Technik dürfe nicht zum Antipoden der Sinnlichkeit werden, wenn es darum gehe, eine bestimmte Atmosphäre herzustellen. Er führte in diesem Zusammenhang die Skulptur Der Hund von Alberto Giacometti an, dessen einzigartige Wirkung der Künstler dadurch zustande gebracht habe, dass er beim Erschaffen dieses Werks nach eigenen Worten «ganz Hund» gewesen sei.

\section{Geistiger Knabberstoff}

Über ein sehr entspanntes Verhältnis zur Technik wies sich Anton Gunzinger aus, vielleicht nicht zuletzt des-

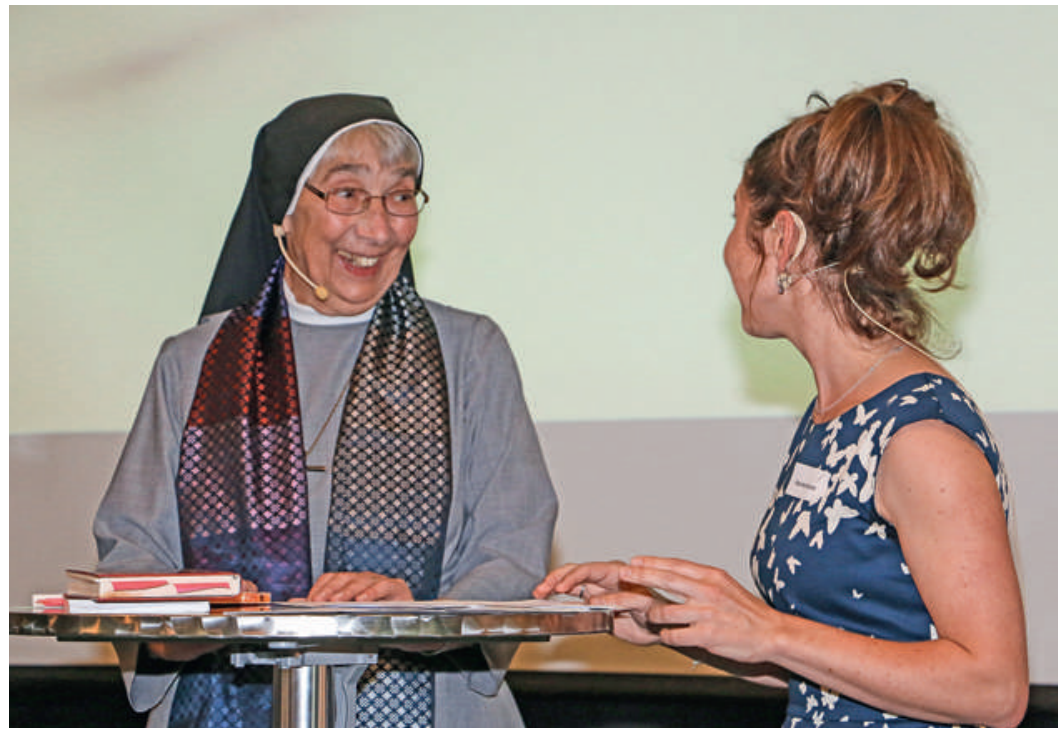

Schwester Liliane Juchli im Gespräch mit Maya Karin Arnold.

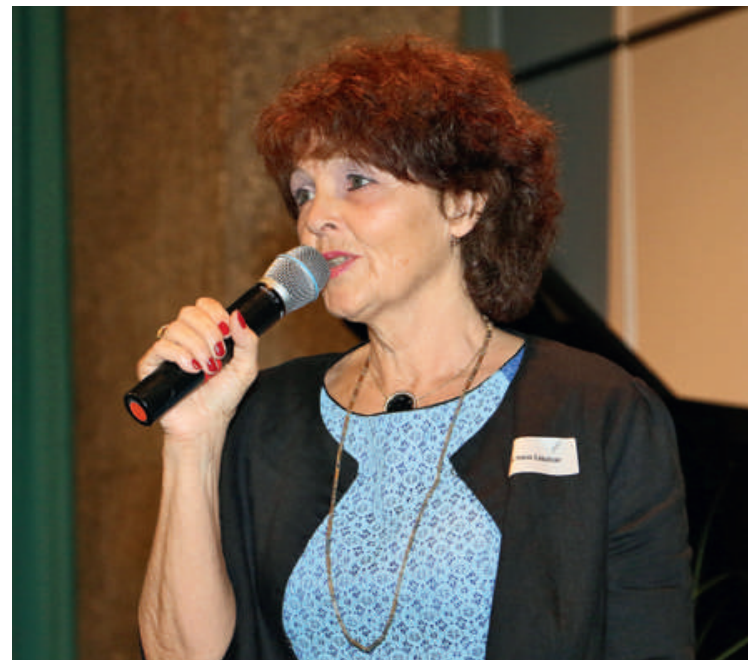

Annina Hess von der Akademie Menschenmedizin moderierte das Symposium.

halb, weil er als Entwickler eines "Supercomputers» unbestrittenermassen etwas davon versteht. Auch aus seiner eigenen Praxis führte er eine Reihe von Beispielen faszinierender und zweifellos sehr sinnvoller Anwendungen der Computertechnologie an. Ebenso aufschlussreich war es, aus seinem berufenen Mund den "Mythos von der Technik, die stark und frei macht», relativiert zu hören. Er erinnerte in amüsanter Weise daran, dass ein wesentlicher Unterschied zwischen Mensch und Computer darin bestehe, dass Letzterem das Ich-Bewusstsein fehle. Mit Maschinen zu kommunizieren, sei einfach nicht spannend, ja eigentlich sogar langweilig, meinte er augenzwinkernd. Im Übrigen sei auch die Naturwissenschaft nicht immer eindeutig: «Je nachdem, wie man die Frage stellt, lautet die Antwort anders."

Den Schlusspunkt setzte Ludwig Hasler, der in der Akademie Menschenmedizin den Status eines Hausphilosophen innehat. Sein Auftritt war ein Heimspiel, das er wie gewohnt geistvoll, witzig und mit einem zünftigen Schuss (Selbst-)Ironie absolvierte. Er kommentierte die Technik-Ambivalenz, die für das Symposium kennzeichnend war, in der ihm eigenen trocken-brillanten Rhetorik und sorgte damit ebenso für Erheiterung wie für zusätzliche Einsichten. Mit Sätzen wie «Was den Menschen ausmacht, ist die Lücke, durch die die Person wirkt» gab er dem Publikum gleich noch etwas geistigen Knabberstoff mit auf den Heimweg.

Der Tagungsband mit sämtlichen Referaten des Symposiums "Technik - Mensch - Heilkunst» kann online bestellt werden unter www.menschenmedizin.com $\rightarrow$ Symposium-Plattform $\rightarrow$ Tagungsband. 